\title{
AS PESQUISAS SOBRE CIÊNCIA, TECNOLOGIA E SOCIEDADE NO NORDESTE: UM RETRATO DAS DISSERTAÇÕES NA ÁREA DE ENSINO DE CIÊNCIAS NATURAIS E MATEMÁTICA
}

\author{
Paulo Gonçalo Farias GonÇAlves ${ }^{1}$ E Gilberlândio Nunes da Silva ${ }^{2}$ \\ ${ }^{1}$ Universidade Federal do Cariri - UFCA \\ ${ }^{2}$ Universidade Estadual da Paraíba - UEPB \\ <paulo.goncalo@ufca.edu.br>. <gil.gilberlandionunes@gmail.com>
}

DOI: 10.21439/conexoes.v10i4.1023

\begin{abstract}
Resumo. Surgindo como um movimento desencadeador de reflexões acerca das relações entre a sociedade e o desenvolvimento científico e tecnológico, a CTS coloca-se como uma perspectiva promissora para a educação. Por constituir-se como uma abordagem em potencial para o ensino de Ciências Naturais e Matemática, o presente artigo tem o intuito de apresentar um retrato das pesquisas que abordaram a perspectiva CTS, desenvolvidas no âmbito de Programas de Pós-graduação de Instituições de Ensino Superior do Nordeste. Constituindo-se como uma pesquisa estado da arte, selecionou-se 10 dissertações desenvolvidas em programas com nota igual ou superior a 4. As pesquisas selecionadas foram categorizadas em função das áreas do conhecimento e dos enfoques das pesquisas. Em relação às áreas do conhecimento, apesar dos trabalhos estarem distribuídos em três dos quatro campos que compõem a área de Ensino de Ciências Naturais e Matemática, ainda é pequeno ou inexistente o número de investigações em Educação Matemática ou Ensino de Biologia. No que se refere aos enfoques das pesquisas, identificou-se que os trabalhos visaram, sobretudo, desenvolver experiências formativas na educação básica ou na formação de professores alinhadas a temas que emergiam da realidade dos sujeitos das pesquisas. Torna-se necessária uma maior difusão da abordagem CTS no âmbito das Pós-graduações e da educação básica, visando contribuir para o debate acerca da necessidade de um desenvolvimento científico e tecnológico alinhado com os anseios e o bem-estar da humanidade e do meio ambiente.
\end{abstract}

Palavras-chaves: Ensino de Ciências Naturais e Matemática; Abordagem CTS; Estado da Arte.

\begin{abstract}
The STS (Science, Technology and Society) is constituted as a potential approach to the teaching of Natural Sciences and Mathematics since it emerged as a trigger movement of reflections on the relationship between society and the scientific and technological development, putting up a promising perspective for education. This article aims to present a report of research that addressed the STS perspective developed in the scope of the graduate program of higher education institutions from Brazilian northeast. Establishing itself as a search state of the art, it selected 10 dissertations developed programs with grade equal to or greater than 4 . The selected studies were categorized according to fields of knowledge and approaches of research. Regarding the areas of knowledge, despite the work being distributed in three of the four fields that make up the area of Natural Science and Mathematics Education, the number of research in mathematics education or biology teaching is still little or inexistent. With regard to approaches of research, we identified that the work aimed, above all, develop formative experiences in basic education or in teachers training aligned to themes that emerged from the reality of the subjects of the research. It is necessary for wide dissemination of STS approach within the Postgraduate and basic education, to contribute to the debate about the need for scientific and technological development associated with humanity wishes and welfare and the environment.
\end{abstract}

Keywords: Education of Natural Sciences and Mathematics. CTS approach. State of art. 


\section{INTRODUÇÃO}

Historicamente o final da década de setenta do século passado, foi marcado pela busca de melhorias para o ensino de ciências, estas estiveram vinculadas aos processos de produção e divulgação de conhecimento científico, e ainda de forma modesta se preocupa com os avanços das pesquisas sobre a didática das ciências, e atualmente, o ensino de ciência na educação básica e superior ainda vem incorporando ideias da perspectiva CTS das décadas de 1950, 1960 e 1970. É importante pontuar que o desenvolvimento científico depende de inovações veiculadas aos avança da ciência das tecnologias e suas aplicações na educação, a fim de resolver problemas de ordem social, político, econômico e ambiental, que são provocados pelo descontrolo das atividades científicas e tecnológicas (KRASILCHIK, 2000).

Nesse contexto, na década de 70 surgem no campo educacional os estudos CTS (Ciência, Tecnologia e Sociedade) associados ao ensino de ciências, com a proposição de novos currículos que buscassem incorporar conteúdo de CTS. Nesse sentindo, diversos materiais didáticos para o ensino de ciências foram produzidos com enfoque CTS para todos os níveis educacionais, desde o Ensino Fundamental à Graduação em diversos países, sobretudo nos Estados Unidos, Canadá e na Europa.

No Brasil, preocupações com a inclusão no currículo de implicações sociais da ciência já eram percebidas, por exemplo, nos materiais desenvolvidos pelo Centro de Ciências do Estado de São Paulo nos anos 70, porém pesquisas e desenvolvimento mais sistemático de materiais com a denominação CTS começaram a surgir somente no final dos anos 90 (SANTOS et al. 2010).

Nessa perspectiva, o ensino de ciências necessita urgentemente que as universidades brasileiras ofereçam cursos de formação inicial e continuada de qualidade e que possibilitem a inserção dos currículos CTS nos processos didáticos, bem como suas aplicações nas escolas públicas de educação básica, nesse sentido, as instituições de ensino superior não pode formar professor que não atenda as prescrições dos documentos curriculares oficiais da educação básica.

Assim, a educação básica e superior não pode estar presa ao modelo transmissão - recepção, no qual temos o professor como o sujeito que possui a fonte do saber e o aluno como o sujeito que recebe as informações transmitidas. Esse fato tem favorecido a falta de contextualização no ensino de Ciências, caracterizado pela transmissão de fórmulas, leis e teorias, mostrando um ensino desvinculado com as ações sociais dos estudantes, o que desmotiva a aprendizagem desta ciência.
Nos documentos curriculares Nacionais (BRASIL, 2001, BRASIL, 2002; BRASIL, 2006) o conhecimento das ciências deve considerar o contexto sociocultural a qual os estudantes estão inseridos de maneira que, os mesmo insiram o conhecimento disciplinar nos diferentes setores da sociedade e relacione com os aspectos políticos, sociais e tecnológicos de cada época.

Diante dessas questões, o presente estudo tem o intuito de apresentar um retrato das pesquisas que abordaram a perspectiva CTS, desenvolvidas no âmbito de Programas de Pós-graduação na área de Ensino de Ciências Naturais e Matemática de Instituições de Ensino Superior da região Nordeste.

\section{FUNDAMENTAÇÃO TEÓRICA: Considera- ções sobre o enfoque CTS no ensino de Ci- ências}

Historicamente movimento educativo CTS surgiu nos anos 60 e 70 no meio universitário, e nos anos 80 no Ensino Médio, no cenário internacional. Atualmente tem como objetivo, a alfabetização científica na perspectiva da construção da cidadania, de maneira que esses se tornem aptos a participar dos processos de tomada de decisão relacionados á Ciência e Tecnologia (DANTAS FILHO; SILVA G. N.AND SILVA, 2015; MEMBIELA, 1997). Assim, o ensino CTS veio "substituir o currículo convencional de ciências, visando implantar um currículo centrado no desenvolvimento de conhecimentos e atitudes úteis para a vida diária dos educandos" (REIS; $\mathrm{aO}, 2008$, p.767).

Neste contexto, segundo Santos e Mortimer (2000) o objetivo central do ensino CTS na educação básica é promover a educação científica e tecnológica dos cidadãos. Para Santos e Schnetzler (1997) este deve auxiliar o aluno na construção de conhecimentos, habilidades e valores necessários para tomar decisões responsáveis sobre questões de ciência e tecnologia na sociedade. Corroborando com estes autores Angotti e Auth (2001), Cruz e Zylbersztajn (2001); sinalizam que este ensino oportunizam os alunos a atuarem na solução de problemas do seu contexto, ampliando a cultura científica e a capacidade de dialogar sobre questões sócias científicas (TEIXEIRA, 2003).

Nas últimas décadas, são significativas as discussões no do ensino de ciências, mostrando a necessidade de uma formação básica com qualidade, e o enfoque CTS sinaliza para tal educação com a preparação profissional e para o acesso ao ensino superior (DANTAS FILHO; SILVA G. N.AND SILVA, 2015). Segundo Santos et al. (2010) o aumento dos problemas sociais no mundo favoreceu a implantação do currículo CTS, bem como com a incorporação de temas socioambientais e 
AS PESQUISAS SOBRE CIÊNCIA, TECNOLOGIA E SOCIEDADE NO NORDESTE: UM RETRATO DAS DISSERTAÇÕES NA ÁREA DE ENSINO DE CIÊNCIAS NATURAIS E MATEMÁTICA

suas relações entre Ciência, Tecnologia e Sociedade.

Movimento CTS deu início ao questionamento da autonomia e da tecnociência, destacando a não neutralidade e ressaltando que o progresso tecnocientífico nem sempre propaga melhorias sociais. Segundo Avellaneda e Vonlisingen (2011), podemos ressaltar que o movimento CTS demonstrou que os investimentos na área de CT são carregados de interesses particulares, econômicos, políticos e que nem sempre seus avanços e benesses estarão disponíveis a toda a população.

Corroborando com o pensamento de Rachel Carson, publicado no livro Primavera Silenciosa, o que apresentou fortes influências ao surgimento do movimento CTS foi à conscientização pública de que a natureza era vulnerável a intervenção humana, nesse sentido, surgiu à necessidade de regularizar produção industrial para proteger o meio ambiente. A obra de Carson foi considerada uma das principais bases para o pensamento ambiental, tornando-se um referencial no debate em torno dos desequilíbrios ecológicos provocados pela ação humana (JACOBI, 2005).

Em linhas gerais, o movimento CTS constitui uma crítica à concepção tradicional de ciência e tecnologia, buscando recolocar as questões sociais no centro do processo de desenvolvimento científico e tecnológico, dessa forma quando CTS adentra o Ensino de Ciências, busca antes de tudo alterar a relação tradicional e linear existente entre professor e aluno, colocando ambos como parceiros na construção do conhecimento científico (DANTAS FILHO; SILVA G. N.AND SILVA 2015).

O movimento CTS é destacado por tradições diferentes, A Norte-americana e a europeia. Segundo $\mathrm{Au}-$ ler (2002) a norte-americana foi marcada por protestos ecológicos contra a bomba atômica e o uso de produtos químicos já a europeia de acordo com Carletto e Pinheiro (2010) afirmam que esta se norteou em trabalhos da sociologia clássica do conhecimento e nas reflexões de Thomas Kuhn Avellaneda e Vonlisingen (2011). De acordo com Vaz, Vaz, FAGUNDES e PINHEIRO (2009), a tradição europeia originou-se por volta de 1979 no "Programa Forte", cujos autores foram Barry Barnes, David Bloor e Steven Shapin, como uma tradição de investigação acadêmica.

No Brasil, o movimento CTS encontra outras correntes educacionais como a educação problematizadora e dialógica de Paulo Freire. Na visão de Auler (2002) as concepções freirianas apresentam semelhanças com o que Cachapuz denominou Pós-mudança conceitual, podendo sinalizar caminhos no contexto brasileiro para o ensino de Ciências. Além disso, o autor defende a reinvenção da concepção freiriana que deve abranger uma visão mais crítica das interações CTS, o que segundo este é fundamental para a leitura do mundo contemporâneo (CONTIER, 2009; QUADROS; LELIS; FREITAS, 2015).

A conexão estabelecida entre a sociedade e a ciência, vinculadas aos conteúdos científicos estudados é relevante para a compreensão de situações problemas do cotidiano e favorecem ao aluno a assimilação de conhecimento (KRASILCHIK, 2000).

Neste contexto, ensino de Ciências Naturais vem ganhando espaço no cenário nacional, com a preocupação de como deve ocorrer o processo de construção de conhecimento. Essa visão se apoia nos documentos oficiais, os quais apontam para o desenvolvimento de alunos e professores comprometido com uma educação básica de qualidade, que desenvolva competências e habilidades nos sujeitos envolvidos no processo, e estes compreendam os aspectos relacionados ao contexto sociocultural do estudante com a interação professor/estudantes/conhecimento (SANTOS et al., 2010) já Dantas Filho (2015) sinaliza para um diálogo entre as ideias previas dos estudantes com a sua visão científica e a mediação do professor, construindo junto o conhecimento científico (DANTAS FILHO; SILVA G. N.AND SILVA, 2015).

A contextualização da ciência e da tecnologia é uma dos principais objetivos do movimento CTS na educação básica, assim como a promoção da participação pública nos processos e nas decisões relacionados à educação (LÓPEZ-CEREZO, 2009). Segundo Santos et al. (2010) o enfoque CTS pode ser abordado em qualquer nível de ensino, no entanto é mais comum no Ensino Médio e no superior. Ainda Santos et al. (2010) afirma que educar em uma perspectiva CTS é aproximar a ciência da vida das pessoas, inserindo-as em contexto de crítica e argumentação em relação à forma como a ciência e a tecnologia têm interferido de alguma forma na vida de todos (MAGALHÃES; TENREIRO-VIEIRA, 2006).

As orientações CTS são defendidas no ensino de ciências quando é possível fazer demonstrações relacionadas aos fenômenos científicos existentes, vinculados a tecnologia e a vida social dos alunos para torná-los cientificamente alfabetizados. Nessa perspectiva, Santos et al. (2010) aponta que o enfoque CTS como proposta educativa visa proporcionar formação em conhecimentos e valores que favoreçam a participação cidadã nas inovações tecnocientíficas, bem como na capacitação dos envolvidos para a tomada de decisões responsáveis considerando a sociedade e o meio ambiente, assim como as dimensões afetivas éticas e culturais (MELLO; GUAZELLI, 2011). 
Nesse sentindo, ao optar trabalhar com CTS, deve desenvolver atividades junto aos educandos de forma dialógica, problematizando os conteúdos para que os alunos possam tomar consciência de sua participação no mundo, bem como viabilizar aos alunos a aquisição de valores éticos, assim como a capacidade de argumentação, e a criticidade diante da necessidade de buscar soluções para os problemas do seu dia a dia (SANTOS; SCHNETZLER, 1997).

\section{METODOLOGIA}

A presente investigação consiste numa pesquisa do tipo estado da arte. O estado da arte é um tipo particular de pesquisa bibliográfica que tem como enfoque mapear os estudos de uma determinada área do conhecimento, visando sistematizar e avaliar as características, tendências e lacunas das produções ligadas a uma temática específica dessa área. Em relação ao tema escolhido para realização da presente pesquisa, nos propusemos a analisar trabalhos que versavam sobre Ciência, Tecnologia e Sociedade (CTS) no ensino de Ciências Naturais e Matemática.

Discutindo sobre a importância das investigações estado da arte, Romanowski e Ens (2006, p.38-39) afirmam que:

\footnotetext{
O interesse por pesquisas que abordam 'estado da arte' deriva da abrangência desses estudos para apontar caminhos que vêm sendo tomados e aspectos que são abordados em detrimento de outros. A realização destes balanços possibilita contribuir com a organização e análise na definição de um campo, uma área, além de indicar possíveis contribuições da pesquisa para com as rupturas sociais. A análise do campo investigativo é fundamental neste tempo de intensas mudanças associadas aos avanços crescentes da ciência e da tecnologia.
}

Nesse sentido, o mapeamento de aspectos inerentes ligados a uma determinada área do conhecimento, pode contribuir tanto ao pesquisador iniciante, por apresentar um panorama geral das investigações já desenvolvidas; quanto ao pesquisado experiente, por auxiliá-lo na identificação de novas perspectivas a serem exploradas.

Para o desenvolvimento dessa pesquisa, adotamos os procedimentos metodológicos propostos por (ROMANOWSKI, 2002), a saber: 1) Definir descritores de busca; 2) localizar banco de trabalhos; 3) estabelecer critérios de seleção dos trabalhos; 4) levantar os trabalhos; 5) coletar os trabalhos; 6) ler os trabalhos; 7) organizar o relatório; 8) analisar e elaborar conclusões iniciais.
Definimos como descritores para deflagrar as buscas pelos trabalhos os seguintes termos: "Ciência, Tecnologia e Sociedade" e sua abreviação, "CTS". Esses dois descritores foram empregados nas seções de Dissertações e Teses, disponíveis nos sites dos programas de pós-graduação stricto sensu da área de Ensino de Ciências Naturais e Matemática ofertados por Instituições de Ensino Superior (IES) no Nordeste.

Visando estabelecer o corpus de textos, adotamos como critério de seleção dos trabalhos restringir as buscas por pesquisas desenvolvidos em programas de pósgraduação com nota igual ou superior a 4. A Tabela 1 a seguir apresenta a lista de IES em que foram realizadas as buscas e o número de trabalhos selecionados:

Tabela 1: Lista de programas de pós-graduação e número de trabalhos selecionados.

\begin{tabular}{c|l|c}
\hline $\begin{array}{l}\text { SIGLA } \\
\text { IES }\end{array}$ & Programa & $\begin{array}{c}\mathrm{N}^{\circ} \text { trabalhos } \\
\text { selecionados }\end{array}$ \\
\hline UFBA & $\begin{array}{l}\text { Ensino, Filosofia e } \\
\text { História das Ciências }\end{array}$ & 0 \\
\hline UEPB & $\begin{array}{l}\text { Ensino de Ciências e } \\
\text { Matemática }\end{array}$ & 3 \\
\hline UFRPE & Ensino das Ciências & 1 \\
\hline UFPE & $\begin{array}{l}\text { Educação Matemática e } \\
\text { Tecnológica }\end{array}$ & 0 \\
\hline UFRN & $\begin{array}{l}\text { Ensino de Ciências } \\
\text { Naturais e Matemática }\end{array}$ & 6 \\
\hline UFRN & $\begin{array}{l}\text { Ensino de Ciências e } \\
\text { Matemática }\end{array}$ & 0 \\
\hline
\end{tabular}

Composto por 10 dissertações, o corpus da presente pesquisa foi coletado por meio das bibliotecas eletrônicas de cada IES ou por meio de correio eletrônico, através de contatos os Programas de Pós-graduação. A Tabela 2 seguinte apresenta a lista de dissertações analisadas:

Para fins de organização, análise e discussão dos dados, as dissertações foram lidas e relidas, visando a impregnação dos conteúdos contidos em cada trabalho (LUDKE; ANDRE, 1986). Desse processo de leitura exaustiva, emergiram as seguintes categorias de análise dos dados: áreas do conhecimento e enfoques das pesquisas. Na seção seguinte serão apresentados os resultados da presente investigação. 
AS PESQUISAS SOBRE CIÊNCIA, TECNOLOGIA E SOCIEDADE NO NORDESTE: UM RETRATO DAS DISSERTAÇÕES NA ÁREA DE ENSINO DE CIÊNCIAS NATURAIS E MATEMÁTICA

Tabela 2: Lista de dissertações selecionadas.

\begin{tabular}{|c|c|c|}
\hline $\begin{array}{l}\text { SIGLA } \\
\text { IES }\end{array}$ & Título da Dissertação & Autor \\
\hline UEPB & Vídeo e ensino de Ciências: um olhar CTS sobre a produção dos alunos & Oliveira (2010) \\
\hline UEPB & Cinema e o ensino de Física: uma experiência sob olhar CTS & Mota (2011) \\
\hline UEPB & $\begin{array}{l}\text { Abordagem CTS e ensino de Matemática Crítica: um olhar sobre a formação } \\
\text { inicial dos futuros docentes }\end{array}$ & Silva (2012) \\
\hline UFRN & $\begin{array}{l}\text { Abordando as Relações CTSA no ensino da Química a partir das crenças e } \\
\text { atitudes de licenciandos: uma experiência formativa no sertão nordestino }\end{array}$ & Nunes (2010) \\
\hline UFRN & $\begin{array}{l}\text { O uso da abordagem CTSA no ensino de Energia tendo o desenvolvimento } \\
\text { sustentável como eixo temático }\end{array}$ & Lima Neto (2012) \\
\hline UFRN & $\begin{array}{l}\text { Açude do Cais: uma proposta de utilização de material didático com enfoque } \\
\text { CTS }\end{array}$ & Marques (2014) \\
\hline UFRN & Biogás: discutindo as relações CTS com os alunos do ensino médio & Macedo (2014) \\
\hline UFRN & Um estudo sobre o ensino de evaporação no contexto CTS & Bezerra (2014) \\
\hline UFRPE & $\begin{array}{l}\text { A implementação de uma Abordagem CTS (Ciência-Tecnologia-Sociedade) no } \\
\text { ensino de Química: um olhar sobre a prática docente }\end{array}$ & Firme (2007) \\
\hline
\end{tabular}

\section{RESULTADOS E DISCUSSÃO}

Analisando dissertações publicadas em Programas de Pós-Graduação na área de Ensino de Ciências Naturais e Matemática com base nas áreas do conhecimento que cada pesquisa tomou como enfoque, obtivemos os seguintes resultados, mostrados na Figura 1

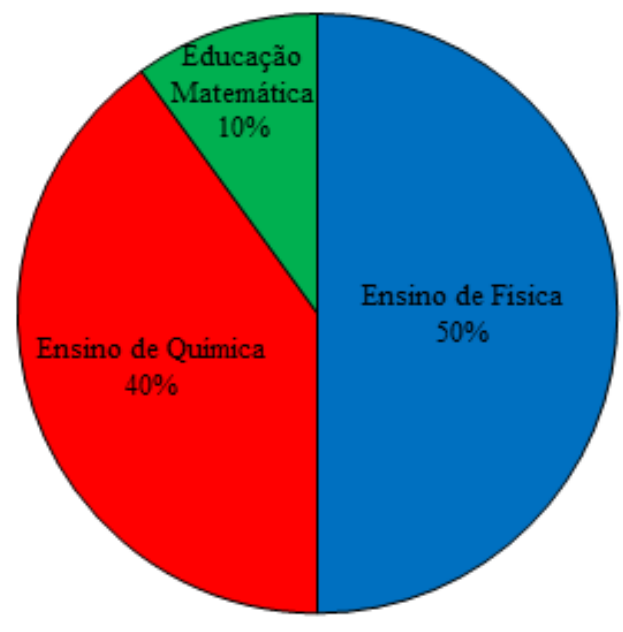

Figura 1: Distribuição os trabalhos em função das áreas do conhecimento.

Como se pode notar, há uma concentração dos trabalhos nas áreas de Ensino de Física e Ensino de Quí- mica $(90 \%)$. Em contrapartida, verifica-se apenas uma pesquisa na área de Educação Matemática (10\%) e nenhuma em Ensino de Biologia. No que se refere ao enfoque das pesquisas, podemos classificar as pesquisas nas seguintes subcategorias: temas gerais (40\%), vários conteúdos simultâneos (20\%), CTS no ensino (20\%) e conteúdos específicos $(10 \%)$. As pesquisas que optaram por temas gerais tiveram como enfoque o tratamento de assuntos transversais. Abordando o tema água para elaboração e aplicação de sequências didáticas, as dissertações tiveram como enfoque discutir questões emergentes do contexto dos participantes da pesquisa, tais como: o processo de degradação do rio ApodiMossoró (NUNES, 2010), a qualidade da água fornecida no município de Cuité-PB (MARQUES, 2014) e as relações entre o tema Evaporação e a constante escassez de água na cidade de Santa Cruz-RN (BEZERRA. 2014).

Quadros (2004) aponta que o tema água pode ser bem explorado e contextualizado no Ensino de Química, pois é possível trabalhar com aspectos conceituais e sócio científicos, através de conteúdos como lei de conservação das massas, ligações químicas, estados físicos da matéria, energia envolvida nas ligações, soluções, reações químicas, velocidade das reações, concentração, etc.

Além disso, abordagem de tal tema no universo da sala de aula, e em particular no Ensino de Ciências, está 
relacionada ao fato de realizar aproximações entre os conceitos químicos e situações do cotidiano do aluno (DANTAS FILHO; SILVA G. N.AND SILVA, 2015). Através deste tema, o professor poderá problematizar investigar e interpretar situações importantes, de maneira que os conceitos químicos contribuam na compreensão e resolução de problemas que estão dentro do contexto dos estudantes de forma crítica e construtiva (BRASIL, 2002).

Discutindo a temática Terremotos, Oliveira (2012) coletou e analisou relatos de moradores do município de João Câmara-RN sobre convivência com abalos sísmicos na região. Esses dados serviram como base para a elaboração e a aplicação de uma sequência de atividades sobre o tema. Outro tema geral abordado foi o Biogás e suas relações com a sustentabilidade, trabalhado por Macedo (2014) a partir do desenvolvimento de uma experiência educacional com alunos do ensino básico.

Nesta perspectiva, fazer educação através de problemas da realidade significa colocar a ciência a serviço da vida, buscando trabalhar os conteúdos não de forma isolada, mas integrá-los a realidade e o contexto de vida dos alunos, para que estes adquiram a capacidade de compreender a natureza (CHASSOT, 2004). "Nos dias atuais, oferecer uma educação para o exercício da cidadania é função primordial das políticas públicas educacionais, conforme estabelece a constituição brasileira e a legislação de ensino" (BRASIL, 2002).

As dissertações que desenvolveram propostas com enfoque em vários conteúdos simultâneos empregaram como recursos didáticos as Tecnologias de Informação e Comunicação. Desenvolvendo atividades voltadas para os conteúdos: Hidrostática e Gravitação; Dantas (2011) utilizou filmes como estratégia para o ensino de Física. Já Oliveira (2010) optou por inserir no âmbito escolar o processo de produção de vídeos educativos pelos alunos, que abordaram temas relacionados aos conteúdos: Mecânica, Dinâmica e Óptica Geométrica.

Morais (2006) destaca as potencialidades do vídeo no ensino de ciências, pode servir para introduzir um novo assunto, servindo de estímulo e motivação, permitem adentrar em realidades de dimensões microscópicas, em situações mais abstratas que não se poderiam ser representadas no quadro de giz. Leite (2015), Silva, Machado e Tunes (2010) afirmam que o vídeo é usado como recursos de simulações práticas de conteúdos distintos e disciplinas curriculares diferentes, pode utilizar para representar os sistemas e suas evoluções e assim diminuir a abstração necessária para compreender os conteúdos e a integração destes recursos serve para le- var o aluno a uma leitura crítica do mundo, colocando-o em diálogo com diversos assuntos e discursos de forma crítica.

Em relação a subcategoria CTS no ensino, os trabalhos classificados tiveram o intuito de propiciar experiências no âmbito da formação de professores com o aporte da Ciência, Tecnologia e Sociedade e suas relações com o Ensino de Ciências Naturais e Matemática. Na pesquisa de Firme (2007), o enfoque foi analisar as concepções de professores sobre CTS e a utilização dessa perspectiva pelos docentes durante suas aulas. Em Silva (2012), a ideia foi analisar as contribuições de uma proposta formativa sobre a abordagem CTS na capacitação de professores de Matemática. Nesse contexto, Santos et al. (2010) afirma que a formação docente deve oferecer uma educação para o exercício da cidadania e é função primordial das políticas públicas educacionais, conforme estabelece a constituição brasileira e a legislação de ensino. Essa função vem sendo defendida por muitos professores pesquisadores da educação básica, atribuindo-se as disciplinas de ciências da natureza o papel de proporcionar um ensino crítico, participativo, reflexivo e humano (QUADROS, 2004).

Sendo a única pesquisa que optou por trabalhar com um conteúdo específico, Lima Neto (2012) teve o intuito de desenvolver uma experiência educativa acerca do conceito de energia, tomando como eixo temático o tema desenvolvimento sustentável. Segundo o autor, as seções didáticas desenvolvidas buscaram além de trabalhar o conceito de energia, "[...] compreender suas transformações e lei de conservação, bem como seus processos de produção, distribuição e consumo no contexto das leis da física em que está envolvido" (LIMA NETO, 2012, p.6).

Nesse sentido, (SANTOS et al., 2010) afirma que é importante elaborar propostas de ensino que possam trabalhar temas geradores a partir de conceitos científicos, buscando trazer situações problematizadoras que estão dentro do contexto de vida dos alunos para que ocorra a alfabetização científica desses sujeitos.

Corroborando com esse pensamento, Santos e Mortimer (2000) afirmam que o professor deve levar problemas dessa natureza para a sala de aula, buscando estimular o debate entre os discentes, para que esses sujeitos possam discutir as diferentes soluções e assim possam se posicionar de forma crítica sobre o tema a partir de um ensino que envolva os conteúdos científicos, proporcionando uma aprendizagem significativa e que se enquadre na vida do aluno, ajudando-o a resolver e se posicionar sobre as situações problemas do cotidiano. 
AS PESQUISAS SOBRE CIÊNCIA, TECNOLOGIA E SOCIEDADE NO NORDESTE: UM RETRATO DAS DISSERTAÇÕES NA ÁREA DE ENSINO DE CIÊNCIAS NATURAIS E MATEMÁTICA

\section{CONSIDERAÇÕES FINAIS}

Surgindo como um movimento desencadeador de reflexões críticas acerca das relações entre a sociedade e o desenvolvimento científico e tecnológico, a abordagem CTS coloca-se como uma perspectiva promissora para a educação, e em particular, para o ensino de Ciências Naturais e Matemática.

Considerando a importância da abordagem CTS como uma estratégia a ser inserida no contexto educacional, o presente estudo visou apresentar um retrato das pesquisas desenvolvidas em Programas de PósGraduação da área de Ensino de Ciências Naturais e Matemática vinculados a IES do Nordeste. Para isso, os trabalhos foram categorizados e discutidos em função das áreas do conhecimento que se inseriam e dos enfoques tomados.

Em relação às áreas do conhecimento das pesquisas, apesar dos trabalhos selecionados estarem distribuídos em três dos quatro campos do saber que compõem a área de Ensino de Ciências Naturais e Matemática, torna-se necessária uma ampliação no número de investigações em Ensino de Biologia e em Educação Matemática com tomem como aporte a abordagem CTS.

No que se refere aos enfoques das pesquisas, de um modo geral, identificamos que os trabalhos visaram, sobretudo, propor e empreender experiências formativas no âmbito da educação básica ou da formação de professores alinhadas a temas que emergiam da realidade dos sujeitos de cada investigação.

Além disso, apesar de adotarem temas transversais, diversos ou conteúdos mais específicos, todos com potencialidades para o desenvolvimento de propostas interdisciplinares, as investigações selecionadas se detiveram a apresentar os temas com maior ênfase à perspectiva de uma única disciplina.

Com base no que foi discutido, torna-se necessária uma maior difusão da abordagem CTS no âmbito dos Programas de Pós-graduação e na educação básica, visando contribuir para o debate acerca da necessidade de um desenvolvimento científico e tecnológico realmente alinhado com os anseios e o bem-estar da humanidade e do meio ambiente.

\section{REFERÊNCIAS}

ANGOTTI, J. A. P.; AUTH, M. A. Ciência e tecnologia: Implicações sociais e o papel da educação. Ciência \& Educação, v. 7, n. 1, p. 15-27, 2001.

AULER, D. Interações entre Ciência-TecnologiaSociedade no Contexto da Formação de Professores de Ciências. Tese (Tese) - Universidade Federal de Santa Catarina, Florianópolis, 2002.

AVELLANEDA, M. F.; VONLISINGEN, I. Uma mirada à la educación científica desde los estúdios sociales de la ciência y la tecnologia latino-americanos: abriendo nuevas ventanas para la educación. Alexandria Revista de Educação em Ciência e Tecnologia, v. 4, n. 2, p. 225-246, 2011.

BEZERRA, M. E. B. Um estudo sobre o ensino de evaporação no contexto ciência, tecnologia e sociedade. Dissertação - Ensino de Ciências Naturais e Matemática - Universidade Federal do Rio Grande do Norte, Natal, 2014.

BRASIL. Diretrizes Curriculares Nacionais para a Formação de Professores da Educação Básica em Nivel Superior. Brasília, 2001.

PCN+: Orientações Educacionais

complementares aos Parâmetros Curriculares Nacionais. Brasília, 2002.

Ciências da natureza e suas tecnologias. 2006.

CARLETTO, M. R.; PINHEIRO, N. A. M. Subsídios para uma prática pedagógica transformadora: contribuições do enfoque cts. IENCI, v. 15, n. 3, p. 507-525, 2010.

CHASSOT, a. Para que (m) é útil o ensino?. 2. ed. : Ulbra, 2004.

CONTIER, D. Relações entre ciência, tecnologia e sociedade em museus de ciências. Dissertação (Mestrado) - Faculdade de Educação da Universidade de São Paulo, USP, São Paulo, 2009.

CRUZ, S. M. S. C.; ZYLBERSZTAJN, A. O enfoque ciência, tecnologia e sociedade e a aprendizagem centrada em eventos. In: PIETROCOLA, M. o. (Ed.). Ensino de Física: conteúdo e epistemologia numa concepção integradora. Florianópolis, 2001.

DANTAS FILHO, F. F; SILVA G. N.AND SILVA, H. C. Entendimento da abordagem ctsa no ensino de química e as dificuldades apontadas por professores de escolas da cidade de campina grande - pb em inserir esse enfoque nas suas aulas. ScientiaAmazonia, 2015.

DANTAS, G. M. O cinema e o ensino da Física: uma experiência sob olhar CTS. Dissertação Universidade Estadual da Paraíba, Campina Grande, 2011. 
AS PESQUISAS SOBRE CIÊNCIA, TECNOLOGIA E SOCIEDADE NO NORDESTE: UM RETRATO DAS DISSERTAÇÕES NA ÁREA DE ENSINO DE CIÊNCIAS NATURAIS E MATEMÁTICA

FIRME, R. N. A implementação de uma abordagem CTS (Ciência-Tecnologia-Sociedade) no ensino da química: um olhar sobre a prática pedagógica. Dissertação - Universidade Federal Rural do Pernambuco, Recife, 2007.

JACOBI, P. Educar para a sustentabilidade: Complexidade, reflexividade, desafios. Revista Educação e Pesquisa, v. 31, n. 2, 2005.

KRASILCHIK, M. Reformas e realidade: o caso do ensino das ciências. São Paulo em Perspectiva, v. 14, n. 1, p. 85-93, 2000.

LEITE, B. S. Tecnologias no Ensino de Química: Teoria e Prática na Formação Docente. Curitiba: Appris, 2015.

LIMA NETO, J. A. O uso da abordagem CTSA no ensino de energia tendo o desenvolvimento sustentável como eixo temático. Dissertação (Mestrado) Mestrado em Ensino de Ciências Naturais e Matemática) - Universidade Federal do Rio Grande do Norte, Natal, 2012.

LÓPEZ-CEREZO, J. A. Ciencia, tecnología y sociedade: el estado de la cuestionenla europa y estados unidos. In: OEI, C. de Altos Estudios Universitarios de la (Ed.). Educación, Ciencia, Tecnología y Sociedad. Documentos de Trabajo. 2009. p. $23-26$

LUDKE, M.; ANDRE, M. E. D. A. Pesquisa em educação:abordagens qualitativas. São Paulo: Editora Pedagógica e Universitaria, 1986.

MACEDO, F. L. Abordando as relações CTS e a sustentabilidade no ensino de química: uma proposta de sequência didática a partir do tema biogás. Dissertação - Mestrado em Ensino de Ciências Naturais e Matemática - Universidade Federal do Rio Grande do Norte, Natal, 2014.

MAGALHÃES, S.; TENREIRO-VIEIRA, C. Educação em ciências para uma articulação ciência, tecnologia, sociedade e pensamento crítico. um programa de formação de professores. Revista Portuguesa de Educação, v. 19, n. 2, p. 85-110, 2006.

MARQUES, A. M. Açude do Cais: uma proposta de aplicação de uma sequência de atividades didáticas em um contexto real. Dissertação - Mestrado em Ensino de Ciências Naturais e Matemática - Universidade Federal do Rio Grande do Norte, Natal, 2014.
MELLO, L.; GUAZELLI, I. A alfabetização científica e tecnológica e a educação para a saúde em ambiente não escolar. Revista Brasileira de Educação Ciência e Tecnologia, v. 4, n. 1, p. 22-41, 2011.

MEMBIELA, I. Una revisión delmovimiento educativo ciencia, tecnología, sociedad. Enseanza de las Ciencias, v. 15, n. 1, p. 51-57, 1997.

MORAIS, C. S. Química digital - Recursos digitais no ensino da química: uma experiência no 7 o ano de escolaridade. Dissertação - Mestrado em Multimídia. Faculdade de Ciências, Porto, 2006.

NUNES, A. O. Abordando as relações CTSA no ensino da química a partir das crenças e atitudes de licenciandos: uma experiência formativa no sertão nordestino. Dissertação - Mestrado em Ensino de Ciências Naturais e Matemática - Universidade Federal do Rio Grande do Norte, Natal, 2010.

OLIVEIRA, A. L. P. Uma sequência didática a partir da temática terremotos com ênfase em CTS. Dissertação - Mestrado em Ensino de Ciências Naturais e Matemática - Universidade Federal do Rio Grande do Norte, Natal, 2012.

OLIVEIRA, F. R. G. Vídeo e ensino de ciências: um olhar CTS sobre a produção dos alunos. Dissertação - Mestrado Profissional em Ensino de Ciências e Matemática - Universidade Estadual da Paraíba, Campina Grande, 2010.

QUADROS, A. L. água como tema gerador que conhecimento químico. São Paulo, 2004.

QUADROS, A. L.; LELIS, I. S. S.; FREITAS, M. L. Ações Construtivas em Química Compartilhando Experiências. : Livraria da Fisíca, 2015.

REIS, P.; aO, C. G. Os professores de ciências naturais e a discussão de controvérsias sociocientíficas: dois casos distintos. Revista Electrónica de Enseanza de lasCiencias, v. 7, n. 3, p. 746-772, 2008.

ROMANOWSKI, J. P. As licenciaturas no Brasil: Um balanço das Teses e Dissertações dos anos 90. Tese (Tese) - Faculdade de Educação da Universidade de São Paulo, São Paulo, 2002.

ROMANOWSKI, J. P.; ENS, R. T. As pesquisas denominadas do tipo estado da arte em educação. Diálogo Educacional, v. 6, n. 19, p. 37-50, 2006.

SANTOS, W. L. P.; GALIAZZE, M. C.; JUNIOR, E. M. P.; SOUZA, M. L.; PORTUGAL, S. O enfoque 
CTS e a educação ambiental: Possibilidades de ambientalização da sala de aula de ciências. : Unijuí, 2010 .

SANTOS, W. L. P.; MORTIMER, E. F. Uma análise de pressupostos teóricos da abordagem c-t-s (ciência-tecnologia- sociedade) no contexto da educação brasileira. Ensaio pesquisa em educação em ciências, v. 2, n. 2, p. 133-162, 2000.

SANTOS, W. L. P.; SCHNETZLER, R. P. Educação em Química: Compromisso com a Cidadania. : Editora da Unijuí, 1997.

SILVA, D. J. R. Abordagem CTS e ensino de matemática crítica: um olhar sobre a formação inicial dos futuros docentes. Dissertação - Mestrado Profissional em Ensino de Ciências e Matemática Universidade Estadual da Paraíba, Campina Grande, 2012 .

SILVA, R. R.; MACHADO, P. F. L.; TUNES, E. Experimentar sem medo de errar. : Editora da Unijuí, 2010.

TEIXEIRA, P. M. M. A educação científica sob a perspectiva da pedagogia histórico-social e do movimento cts no ensino de ciências. Ciência \& Educação, v. 9, n. 2, p. 177-190, 2003.

VAZ, C.; FAGUNDES, A.; PINHEIRO, N. O

surgimento da ciência, tecnologia e sociedade (cts) na educação: uma revisão. In: . I Simpósio Nacional de Ciência e Tecnologia. 2009. p. 98-116. 